\title{
Preliminary study of therapeutic effect of parecoxib on severe radiation mucositis and dermatitis
}

\author{
Wei-Hsuan Huang $^{1}$, Shih-Hua Liu ${ }^{1}$, Gwo-Che Huang ${ }^{1}$, Che-Wei Su ${ }^{1}$, Wan-Ju Lee ${ }^{1}$, Fred Yi-Shueh \\ Chen $^{1}$, Yu-Jen Chen ${ }^{1,2,3,4}$ \\ ${ }^{1}$ Department of Radiation Oncology, MacKay Memorial Hospital, Taipei, Taiwan; ${ }^{2}$ Department of Medical Research, Mackay Memorial Hospital, \\ Taipei, Taiwan; ${ }^{3}$ Department of Medical Research, China Medical University Hospital, Taichung, Taiwan; ${ }^{4}$ Department of Nursing, Mackay Junior \\ College of Medicine, Nursing, and Management, Taipei, Taiwan \\ Contributions: (I) Conception and design: YJ Chen; (II) Administrative support: SH Liu; (III) Provision of study materials or patients: YJ Chen, GC \\ Huang; (IV) Collection and assembly of data: WH Huang, CW Su, FYS Chen, WJ Lee; (V) Data analysis and interpretation: WH Huang; (VI) \\ Manuscript writing: All authors; (VII) Final approval of manuscript: All authors. \\ Correspondence to: Yu-Jen Chen MD, PhD. Department of Radiation Oncology, MacKay Memorial Hospital, Taipei, Taiwan, No. 92 Section 2, \\ Chung Shan North Road, Taipei, Taiwan. Email: chenmdphd@gmail.com.
}

\begin{abstract}
Background: Pain and inflammatory lesions are the main symptom and sign of radiotherapy (RT)-induced severe mucositis and dermatitis (RISMAD). Simultaneous treatment for pain and inflammation of RISMAD remains unsatisfactory. We collected the therapeutic information of parecoxib, a cyclooxygenase-2 (COX-2) inhibitor, on RISMAD in clinical observation.

Methods: A cohort of 11 cancer patients underwent radiotherapy between 2016 to 2020 was retrospectively analyzed. Intensity modulated RT was delivered by linear accelerators with various fractionation. Patients with grade 3 or 4 RISMAD and pain were treated by short-term daily administration of parecoxib via intramuscular injection. Severity of the symptoms was graded with CTCAE 4.0.

Results: Total eleven lesions were treated and analyzed. The incidence of mucositis, dermatitis and pain were $90.9 \%, 54.5 \%$ and $63.6 \%$ respectively. For patients with grade 3 or 4 mucositis and dermatitis, all the cases improved to grade 1 after receiving parecoxib (mucositis: $60 \%$ vs. $0 \%, \mathrm{P}<0.001$, dermatitis: $50 \%$ vs. $0 \%, \mathrm{P}<0.001)$. Pain score improved from 5.8 to $0.9(\mathrm{P}<0.001)$ by visual analogue scale. Time to improvement for dermatitis, mucositis and pain was $6.3 \pm 3.1,10.2 \pm 6.9$ and $7.5 \pm 5.1$ days, respectively. The average dose of parecoxib needed for improvement was $40 \mathrm{mg}$ per day for $3.8 \pm 2.0$ days. During the treatment and followup, there was no significant adverse effects noted in this cohort, such as skin rash, gastrointestinal bleeding, altered platelet function or cardiac events.
\end{abstract}

Conclusions: Parecoxib appears to be a safe and effective agent for managing severe RT induced mucositis, dermatitis and accompanied pain.

Keywords: Parecoxib; mucositis; dermatitis; nonsteroidal anti-inflammatory drugs (NSAIDs); cyclooxygenase-2 $(\mathrm{COX}-2)$ inhibitor

Received: 18 June 2020. Accepted: 12 July 2020; Published: 30 December 2020.

doi: $10.21037 /$ tro-20-46

View this article at: http://dx.doi.org/10.21037/tro-20-46

\section{Introduction}

In recent decades, concurrent chemoradiation (CCRT) has been widely accepted in treatment of multiple cancer sites, including those of head and neck and pelvis. Growing evidence showed clinical benefit of CCRT which might be a result as of the following rationales: radiosensitization by chemotherapeutics, advances in radiotherapy (RT) technologies and improvement in toxicity care. Especially, 
rationales supporting adding chemotherapy to radiation other than radiosensitization include enhancing local control, and potentially eliminating metastasis (1). Besides, chemotherapeutics, targeted therapeutic agents, such as epidermal growth factor receptor (EGFR) inhibitors, has been reported beneficial in control of locally advanced head and neck cancers while combined with RT. One phase III study has shown advantages of concurrent cetuximab and RT compared to RT alone in locally advanced head and neck cancer in locoregional control, progression free survival and overall survival (2), but the combination regimen enhanced toxicity and resulted in lower compliance and delay in completing RT course (3).

The mean incidence of mucositis in patient treated with CCRT or RT alone was $80 \%$, and $34-57 \%$ with severity up to grade 3-4 (4). With concurrent chemotherapy, the risk of grade 3 oral mucositis increased about 4 -fold over RT alone (5). Symptoms of mucositis can be relieved with topical anesthetics or opioid analgesics. Radiation-induced dermatitis are seen in $95 \%$ of patients (6) even in the era of intensity modulated RT (IMRT) to head and neck cancer. The side effects usually develop parallel to mucositis around 2-3 weeks after starting treatment with symptomatic progression near the end of treatment (7). As for RT to pelvic malignancy, such as cervical cancer, anal cancer and rectal cancer, the radiation dermatitis and mucositis are commonly noted due to inhomogeneous radiation dose distribution to perineal skin. For example, in cervical cancer treated with CCRT and high-dose rate brachytherapy, $23.9 \%$ patients developed grade 3 or higher diarrhea as one presentations of gastrointestinal mucositis, and $23.5 \%$ of grade 3-4 vulva skin dermatitis (8). Early soft tissue reaction for pelvic RT also starts at 2-3 weeks following initiation of the treatment. Management of the condition usually contains topical moisturizing cream or sitz bath and wound care with appropriate dressing. Oral or intravenous antibiotics may be required in severe cases, and analgesic for grade 3 or greater toxicity warrants assessment for a potential treatment threat. However, the management of RT-induced severe mucositis and dermatitis (RISMAD) and pain remain unsatisfactory.

Nonsteroidal anti-inflammatory drugs (NSAIDs) are one of the most used medications in managing acute pain and inflammation, with cyclooxygenase (COX) enzyme being the major target of NSAIDs (9). Cyclooxygenase-2 $(\mathrm{COX}-2)$ can be induced endogenously and exogenously to various stimulation, including IL-1, IL-6, TNF- $\alpha$ or LPS, and stress. $\mathrm{PGE}_{2}$ is the major metabolic product of COX-2 and elevated $\mathrm{PGE}_{2}$ level along with other prostaglandin increases vascular permeability, lowers the threshold of pain and induces pain by sensitizing nerve ending in both central and peripheral nervous system, which results in an inflammatory response (10). COX-1 on the other hand is associated with metabolic products that have correlation with initiation of acute inflammatory response while COX-2 is responsible for maintaining the inflammatory process (11). Since the inhibition of COX-1 is related to adverse events in GI tracts, selective COX-2 inhibitors are better tolerated than non-selective NSAIDs but with comparable analgesic efficacy $(9,12)$. Administration of steroid is effective in eliminating inflammatory reactions, but the adverse effects and inability to control pain limit its clinical application. Taken together, for RISMAD with pain, the optimal agents might be those capable in both anti-inflammation and pain relief without major toxicity, such as COX-2 inhibitors.

For patients suffering from severe oral mucositis who are unable to swallow, intravenous or intramuscular administration may be better preferred. Among cox-2 inhibitors, parecoxib could be delivered intramuscularly or intravenously with satisfying efficacy in pain relief, short time to onset and long lasting duration(13,14), In addition, it does not interfere with platelet function in either healthy elderly or non-elderly individuals (15), and is well tolerated by healthy elders at risk of upper gastrointestinal mucosal injury (16). Parecoxib can successfully help reduce opioid use in patients suffering from pain (17). In this preliminary study we evaluated the efficacy and side effect of parecoxib on managing RT-related inflammation and induced pain. We present the following article in accordance with the STROBE reporting checklist (available at http://dx.doi. org/10.21037/tro-20-46).

\section{Methods}

\section{Patients and identification}

Data were retrospectively collected from January 2016 to May 2020 in cancer patients treated with RT with or without concurrent systemic therapy experiencing radiation induced dermatitis or mucositis at our institute who received parecoxib injection. Majority of the subjects had been treated with non-selective COX inhibitors, steroids and/or opioids without significant improvement before administration of parecoxib. The patients were evaluated weekly during the RT treatment by the attending radiation oncologist with record of the RT-related toxicity and dose 
and response of parecoxib. Medical records included cancer diagnosis and stage, systemic therapy regimen, age at the time of treatment, acute toxicity, and its management. The patients' subjective sensation of irritation, pain or burning of the lesion were defined by patient's themselves using Visual Analogue scale (VAS) from 0 to 10, meaning no symptoms or worst. The study was approved by MacKay Memorial Hospital Institution Review Board (Approval Number: 20MMHIS195e) and conducted in accordance with the Declaration of Helsinki (as revised in 2013). For the study only involving retrospectively reviewing medical records, informed consent was not required under the approval of Mackay Memorial Hospital IRB.

\section{Treatment}

Patients were treated with IMRT with linear accelerator with various fraction size, including photon or electron beam. Total dose depended on the treatment guidelines or other special consideration with altered fractionation such as aggressive histology. Patients all received education of initial skin care and side effects management. When the patients developed grade 3 to 4 skin and mucosa reactions and failed to respond to non-selective COX inhibitors, steroids and/or opioids, parecoxib was prescribed with $40 \mathrm{mg}$ per day via intramuscular route for 3 to 4 days consecutively in one week. The attending physician would evaluate the response to the treatment along with patient reported outcome and decide whether if administering next course of parecoxib is needed.

\section{Response evaluation for parecoxib}

Objectively, during the weekly examination, RT toxicities including mucositis and dermatitis were recorded and graded as recommended by CTCAE 4.0 (common terminology criteria for adverse event version). Subjective pain score was estimated by using VAS (visual analogue scale) reported by patients.

\section{Statistical analysis}

Descriptive analysis was first performed for categorical demographics, tumor and symptom characteristics. Paired- $t$ test was used for analyzing symptom improvement after intervention. $P$ values $<0.05$ were considered statistically significant. Analysis was performed by IBM SPSS software version 25 (SPSS Inc., Chicago, IL, USA).

\section{Results}

\section{Patient characteristics}

Patient's characteristics underwent RT are presented in Table 1. Between January 2017 to May 2020, there were total 11 patients who received parecoxib for management of radiation induced toxicities for various cancer and histology types. Of them, 7 patients were male (64\%) and 4 were female $(36 \%)$. The patients' median age of 64 years (range, 47-83 years), with all in good performance (ECOG 0-2). Most of them $(81.8 \%)$ received higher radiation dose of greater than $60 \mathrm{~Gy}$.

\section{Result of efficacy}

Total eleven lesions were treated, among the patients there were 7 (63.6\%) treated with CCRT and 4 (36.4\%) received RT alone. There incidence of mucositis, dermatitis and severe pain were $90.9 \%, 54.5 \%$ and $63.6 \%$ respectively. After receiving parecoxib, the improvements were significant in decreasing the severity of the symptoms. Before treatment, most patients had Grade 3 or higher mucositis and dermatitis, and all improved to Grade 1 after receiving parecoxib (mucositis: $60 \%$ vs. $0 \%, \mathrm{P}<0.001$, dermatitis: $50 \%$ vs. $0 \%, \mathrm{P}<0.001$ ) (Figure $1 A, B$ ). As for pain score, the average pain score before treatment was 5.8 , and improved to 0.9 after receiving parecoxib $(\mathrm{P}<0.001)$ (Figure 1C).

We also evaluated the time to improvement for dermatitis and mucositis, which was defined as the time interval for symptoms returning to grade 1 . As for pain score, time to improvement was defined as VAS decreasing to less than 4, commonly known as no pain with no intervention required. Time to improvement for dermatitis, mucositis and pain was $6.3 \pm 3.1,10.2 \pm 6.9$ and $7.5 \pm 5.1$ days, respectively. The average dose of parecoxib needed for improvement was $40 \mathrm{mg}$ per day for $3.8 \pm 2.0$ days (total dose $152 \mathrm{mg}$ ). During the treatment and follow-up, there was no significant adverse effects noted in this cohort, such as skin rash, gastrointestinal bleeding, altered platelet function or cardiac events (Figure 2).

\section{Discussion}

In this preliminary study, parecoxib appears to be a safe and effective agent for managing RT induced mucositis, dermatitis and accompanied pain. Comparing with historical data, we had similar incidence of toxicity of grade 
Table 1 Patients characteristics

\begin{tabular}{|c|c|}
\hline Patient characteristics & Numbers (\%) \\
\hline \multicolumn{2}{|l|}{ Age } \\
\hline$<50$ years & $3(27.3)$ \\
\hline$\geq 50$ years & $8(72.7)$ \\
\hline Median (years) & 64 \\
\hline Mean \pm SD & $62 \pm 13.5$ \\
\hline Range (years) & $47-83$ \\
\hline \multicolumn{2}{|l|}{ Gender } \\
\hline Male & 7 (63.6) \\
\hline Female & $4(36.4)$ \\
\hline \multicolumn{2}{|l|}{ ECOG } \\
\hline $0-2$ & $11(100.0)$ \\
\hline $3-4$ & $0(0.0)$ \\
\hline \multicolumn{2}{|l|}{ Locations } \\
\hline Head and neck & $8(72.7)$ \\
\hline Gynecology & $1(9.1)$ \\
\hline Gastrointestinal & $2(18.2)$ \\
\hline \multicolumn{2}{|l|}{ CCRT } \\
\hline Yes & $7(63.6)$ \\
\hline No & $4(36.4)$ \\
\hline \multicolumn{2}{|l|}{ Radiation dose (Gy) } \\
\hline$<50$ & $1(9.1)$ \\
\hline $50-60$ & $1(9.1)$ \\
\hline$>60$ & $9(81.8)$ \\
\hline \multicolumn{2}{|l|}{ Symptoms (incidence) } \\
\hline Dermatitis & $6(54.5)$ \\
\hline Mucositis & $10(90.9)$ \\
\hline Diarrhea & $1(9.1)$ \\
\hline Severe Pain (VAS >5) & 7 (63.6) \\
\hline \multicolumn{2}{|l|}{ Grade } \\
\hline 1 & $5(45.5)$ \\
\hline 2 & $3(27.3)$ \\
\hline 3 & $6(54.5)$ \\
\hline 4 & $2(18.2)$ \\
\hline
\end{tabular}

CCRT, concurrent chemoradiation.

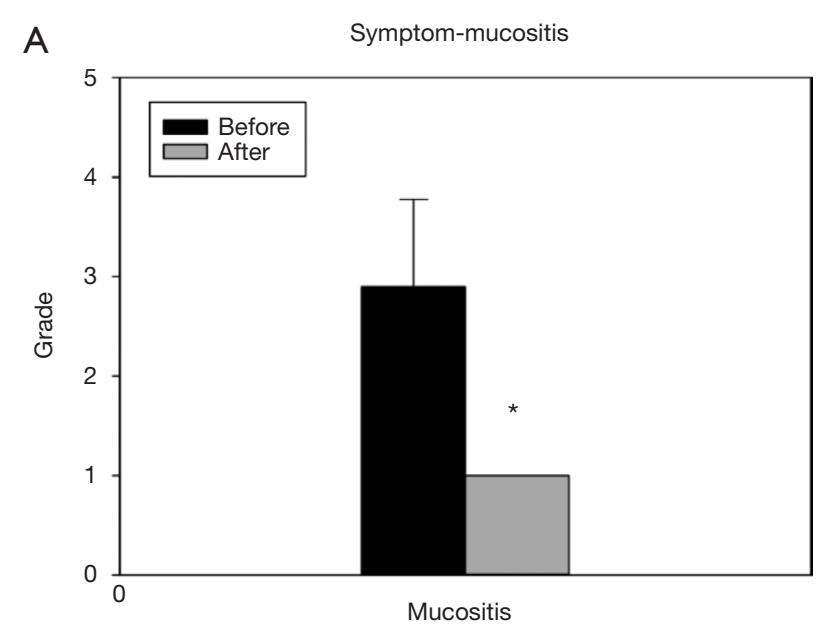

B Symptom-dermatitis

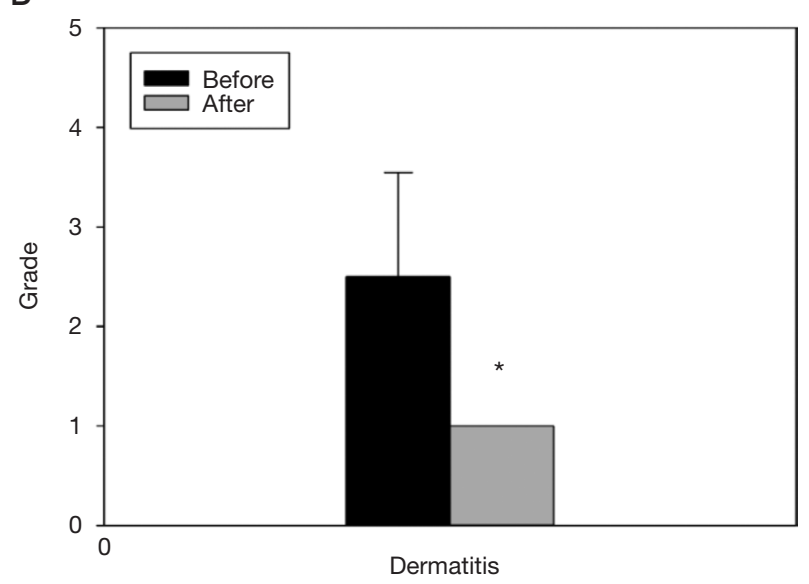

C Symptom-pain

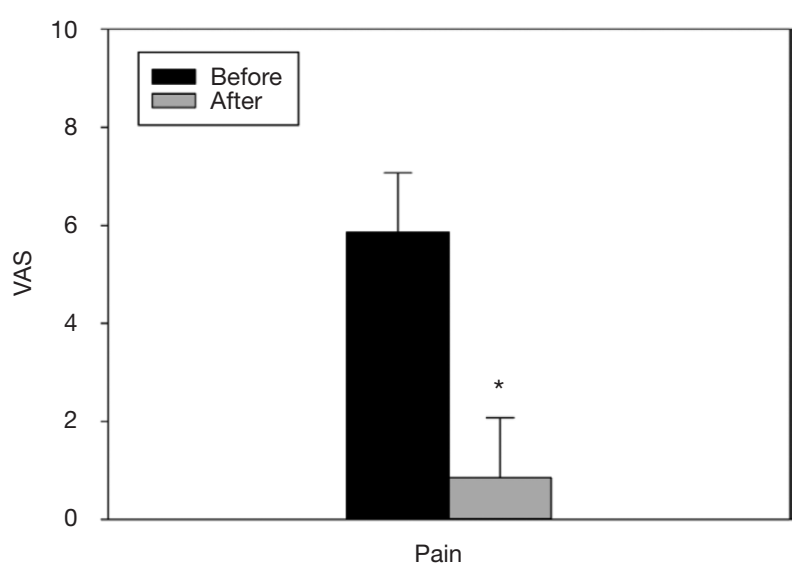

Figure 1 The severity of symptoms of interest were graded with CTCAE 4.0. (A) Mucositis, (B) dermatitis, (C) severe pain. All the above symptoms showed significant improvement after administering parecoxib. *, $\mathrm{P}<0.05$. 


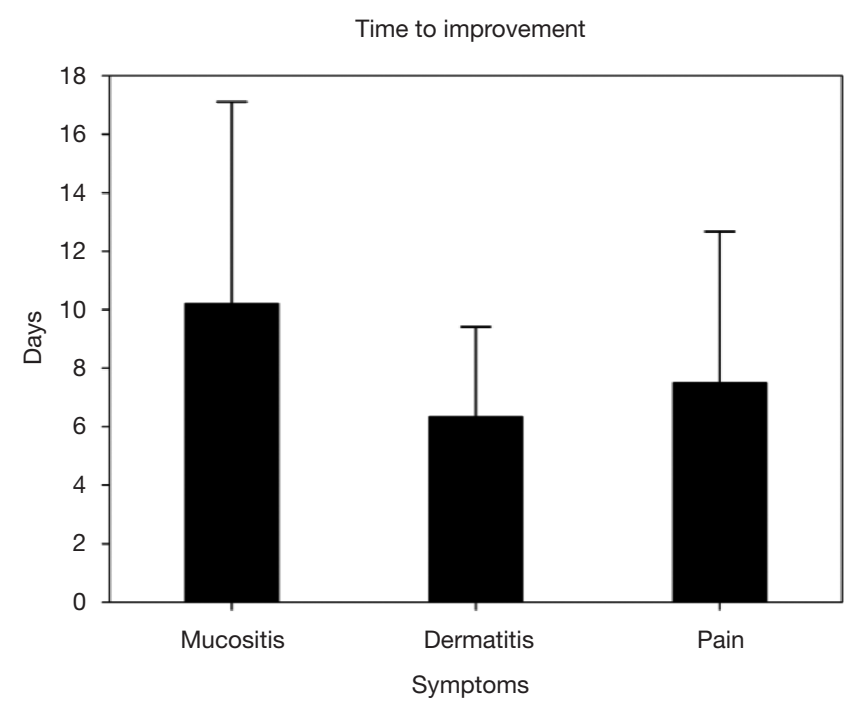

Figure 2 Time to improvement with treatment of parecoxib.

3-4 for either RT alone or CCRT (4). After administering parecoxib, the severity and duration of the symptoms also significantly improved compared to topical treatment (18).

Radiation-induced soft tissue injury, such as RISMAD in this study, has been termed as a "complex wound" because it involves structural damage mediated by groups of free radicals related to DNA damage, and alteration of normal cellular component of functional and structural proteins. Radiation not only interferes with normal maturation, reproduction, and repopulation of epidermal cells, but also targets fibroblast and vasculature (19). Each exposure to radiation leads to inflammatory cell aggregation and direct tissue damage with further impairment of healing by normal tissue (20). Acute radiation injury is a combination of reduced functions of stem cells, inflammation, and epidermal cell necrosis and apoptosis (21).

The mechanism associated with radiation induced skin reaction includes inflammatory response and oxidative stress. After radiation induced cell damage, cells die especially in the form of mitotic death. Late in chronic phase, inflammation and oxidative stress would cause various cytokines, cell cycles, and DNA changes, which sustaining the cascade and lead to late reaction (22). In the initial response, immediately generated inflammatory response is mainly caused by pro-inflammatory cytokines, such as IL-1, IL-3, IL-5 , IL-6 and tumor necrosis factor (TNF)- $\alpha$, chemokines, receptor tyrosine kinase, and adhesion molecules (23). Among them, IL-1 plays an important role in modulating RT induced skin response as shown in mice lacking IL-1 production or receptor develop less inflammation and pathologic changes skin (24). In addition, both IL- 1 and TNF- $\alpha$ are involved in inducing COX-2 expression $(25,26)$.

RT induced dermatitis and mucositis are severe and common complications due to loss of stem cells in the basal layer, compromising the replacement of cells when sloughing. This subsequent denuding leads to mucositis which results in pain and interferes with patient's oral intake of nutrition and daily life. Severe mucositis may lead to $11 \%$ unplanned interruption of treatment, thereby compromising treatment efficacy and patient survival (4). Mucositis also occurs distal to the oropharyngeal cavity along the alimentary tract. When presenting as gastrointestinal mucositis it is commonly known as enteritis or proctitis. Further gastrointestinal mucosal ulceration could lead to perforation, fistula, or abscess formation. Even when the ulcers heal, there could be fibrosis with narrowing of the lumen or obstruction (27). Steroids are well-known for its ability to inhibit all kinds of inflammation, therefore widely used in managing radiation dermatitis and mucositis. With the help of betamethasone, the skin reaction of breast cancer patient receiving adjuvant RT is significantly decreased (28). In patients receiving beclomethasone supplement during radiotherapy treatment, rectal bleeding was reduced but no significant improvement in bowel symptoms at 3 or 12 months (29). Steroids are also known to prolong the treatment time for skin care since they may damage skin's integrity and result in possible further adverse effects which is not suitable for long term use (30). Low dose steroids with equal or greater than $5 \mathrm{mg} / \mathrm{d}$ would also lead to several adverse events such as gastrointestinal bleed or ulcer, infection or fractures (31).

For more specific anti-inflammatory and pain control effect comparing to steroid use, COX inhibitor has excellent anti-inflammatory capacity and pain management allowing reduction in use of opioids (32). In managing mucositis in patients receiving RT dose $>50$ Gy, benzydamine improved grade 3 mucositis from $62.1 \%$ to $36.4 \%$, with reduction in needs of tube feeding, intravenous supplementations (18). Although there were benefits in reducing the severity of mucositis using benzydamine, there was no significant difference in analgesic effect from pain scores comparing the use of sodium bicarbonate alone (33).

Selective COX-2 inhibitor has been shown to reduce skin damage after radiation not only by decreasing production of PGE and PGF2 $\alpha$ to tone down the extent 
of inflammation, but also decreased chemokine mRNA expression in skin tissue, and down-regulate infiltration of monocytes and neutrophils (34). Due to increased COX-2 activity amplifying radiation toxicity in the irradiated area and adjacent normal tissue, increased COX-2 expression in the distant non-irradiated area also increased oxidative stress and DNA damage which may upregulate cancer risk. Activation of COX-2 can enhance resistance of malignant cell to radiotherapy. Hence, inhibition of COX-2 has been proposed for better therapeutic gain and normal tissue amelioration (35). Other RT-related complication such as RT induced neuroinflammation is also found to be associated with an elevation of COX-2 expression and accompanied by increase of PGE2. RT induced changes in vascular permeability is dependent on COX-2 activity, implicating this protein as target for potential therapeutic role in managing side effects on radiation of normal brain tissue (36).

Since it is equipotent comparing with non-selective NSAIDs such as diclofenac but with less undesirable side effects, it would seem more preferable to prescribe selective COX-2 inhibitor (37). Among COX-2 inhibitors, celecoxib is the most studied agent. Though in practice celecoxib showed limited benefits in reducing severity of oral mucositis for head and neck cancer patient (38) or preventing skin toxicity in breast cancer receiving RT (39), there was slight improvement in T-downstaging and $\mathrm{N}$-downstaging for locally advanced rectal cancer (40). Parecoxib, a novel COX-2 inhibitor possessing better pain control (41), also has greater anti-tumor potency than celecoxib (42). In elderly subjects, parecoxib is safe and well tolerated even in multiple dose administration has a decreased risk of gastrointestinal mucosal injury compared to ketorolac (16).

The efficacy of parecoxib on improving pain and RISMAD was evident with a rapid response. The limitations of this study include small sample size and retrospective observation. Based on this preliminary investigation, further study may be designed and carried out for proving its superiority in control of RT-induced mucositis and dermatitis as well as related pain.

\section{Acknowledgments}

Funding: None

\section{Footnote}

Provenance and Peer Review: This article was a standard submission to the journal. The article was sent for external peer review.

Reporting Checklist: The authors have completed the STROBE reporting checklist. Available at http://dx.doi. org/10.21037/tro-20-46

Conflicts of Interest: All authors have completed the ICMJE uniform disclosure form (available at http://dx.doi. org/10.21037/tro-20-46). Yu-Jen Chen serves as an Editorin-Chief of Therapeutic Radiology and Oncology. The other authors have no conflicts of interest to declare.

Ethical Statement: The authors are accountable for all aspects of the work in ensuring that questions related to the accuracy or integrity of any part of the work are appropriately investigated and resolved. The study was conducted in accordance with the Declaration of Helsinki (as revised in 2013). The study was approved by MacKay Memorial Hospital Institution Review Board (Approval Number: 20MMHIS195e). For the study only involving retrospectively reviewing medical records, informed consent was not required under the approval of Mackay Memorial Hospital IRB.

Open Access Statement: This is an Open Access article distributed in accordance with the Creative Commons Attribution-NonCommercial-NoDerivs 4.0 International License (CC BY-NC-ND 4.0), which permits the noncommercial replication and distribution of the article with the strict proviso that no changes or edits are made and the original work is properly cited (including links to both the formal publication through the relevant DOI and the license). See: https://creativecommons.org/licenses/by-nc-nd/4.0/.

\section{References}

1. Seiwert TY, Salama JK, Vokes EE. The concurrent chemoradiation paradigm-general principles. Nat Clin Pract Oncol 2007;4:86-100.

2. Bonner JA, Harari PM, Giralt J, et al. Radiotherapy plus Cetuximab for Squamous-Cell Carcinoma of the Head and Neck. New England Journal of Medicine 2006;354:567-78.

3. Pryor DI, Porceddu SV, Burmeister BH, et al. Enhanced toxicity with concurrent cetuximab and radiotherapy in head and neck cancer. Radiother Oncol 2009;90:172-6.

4. Trotti A, Bellm LA, Epstein JB, et al. Mucositis incidence, severity and associated outcomes in patients with head 
and neck cancer receiving radiotherapy with or without chemotherapy: a systematic literature review. Radiother Oncol 2003;66:253-62.

5. Sanguineti G, Sormani MP, Marur S, et al. Effect of radiotherapy and chemotherapy on the risk of mucositis during intensity-modulated radiation therapy for oropharyngeal cancer. Int J Radiat Oncol Biol Phys 2012;83:235-42.

6. Russi EG, Moretto F, Rampino M, et al. Acute skin toxicity management in head and neck cancer patients treated with radiotherapy and chemotherapy or EGFR inhibitors: Literature review and consensus. Crit Rev Oncol Hematol 2015;96:167-82.

7. Siddiqui F, Movsas B. Management of Radiation Toxicity in Head and Neck Cancers. Semin Radiat Oncol 2017;27:340-9.

8. Chen CC, Lin JC, Jan JS, et al. Definitive intensitymodulated radiation therapy with concurrent chemotherapy for patients with locally advanced cervical cancer. Gynecol Oncol 2011;122:9-13.

9. Lee Y, Rodriguez C, Dionne RA. The role of COX-2 in acute pain and the use of selective COX-2 inhibitors for acute pain relief. Curr Pharm Des 2005;11:1737-55.

10. Mbonye UR, Yuan C, Harris CE, et al. Two distinct pathways for cyclooxygenase-2 protein degradation. J Biol Chem 2008;283:8611-23.

11. McAdam BF, Mardini IA, Habib A, et al. Effect of regulated expression of human cyclooxygenase isoforms on eicosanoid and isoeicosanoid production in inflammation. J Clin Invest 2000;105:1473-82.

12. Padi SS, Jain NK, Singh S, et al. Pharmacological profile of parecoxib: a novel, potent injectable selective cyclooxygenase-2 inhibitor. Eur J Pharmacol 2004;491:69-76.

13. Lloyd R, Derry S, Moore RA, et al. Intravenous or intramuscular parecoxib for acute postoperative pain in adults. Cochrane Database Syst Rev 2009;2009:CD004771.

14. Mateos JL. [Selective inhibitors of cyclooxygenase-2 (COX-2), celecoxib and parecoxib: a systematic review]. Drugs Today (Barc) 2010;46 Suppl A:1-25.

15. Noveck RJ, Laurent A, Kuss M, et al. Parecoxib Sodium does not Impair Platelet Function in Healthy Elderly and Non-Elderly Individuals. Clin Drug Investig 2001;21:465-76.

16. Stoltz R. Upper GI mucosal effects of parecoxib sodium in healthy elderly subjects. Am J Gastroenterol 2002;97:65-71.

17. Malan TP, Marsh G, Hakki SI, et al. Parecoxib Sodium, a Parenteral Cyclooxygenase 2 Selective Inhibitor, Improves
Morphine Analgesia and Is Opioid-sparing following Total Hip Arthroplasty. Anesthesiology 2003;98:950-6.

18. Rastogi M, Khurana R, Revannasiddaiah S, et al. Role of benzydamine hydrochloride in the prevention of oral mucositis in head and neck cancer patients treated with radiotherapy (>50 Gy) with or without chemotherapy. Support Care Cancer 2017;25:1439-43.

19. Goldsmith LA. Physiology, biochemistry, and molecular biology of the skin. Oxford University Press, 1991.

20. Denham JW, Hauer-Jensen M. The radiotherapeutic injury--a complex 'wound'. Radiother Oncol 2002;63:129-45.

21. Bernstein EF, Harisiadis L, Salomon GD, et al. Healing impairment of open wounds by skin irradiation. J Dermatol Surg Oncol 1994;20:757-60.

22. Wei J, Meng L, Hou X, et al. Radiation-induced skin reactions: mechanism and treatment. Cancer Manag Res 2018;11:167-77.

23. Hong JH, Chiang CS, Tsao CY, et al. Rapid induction of cytokine gene expression in the lung after single and fractionated doses of radiation. Int J Radiat Biol 1999;75:1421-7.

24. Janko M, Ontiveros F, Fitzgerald TJ, et al. IL-1 Generated Subsequent to Radiation-Induced Tissue Injury Contributes to the Pathogenesis of Radiodermatitis. Radiat Res 2012;178:166-72.

25. Liu W, Reinmuth N, Stoeltzing O, et al. Cyclooxygenase-2 is up-regulated by interleukin-1 beta in human colorectal cancer cells via multiple signaling pathways. Cancer Res 2003;63:3632-6.

26. Spaziani EP, Benoit RR, Tsibris JC, et al. Tumor necrosis factor-alpha upregulates the prostaglandin E2 EP1 receptor subtype and the cyclooxygenase-2 isoform in cultured amnion WISH cells. J Interferon Cytokine Res 1998;18:1039-44.

27. Galland RB, Spencer J. Spontaneous postoperative perforation of previously asymptomatic irradiated bowel. Br J Surg 1985;72:285.

28. Ulff E, Maroti M, Serup J, et al. A potent steroid cream is superior to emollients in reducing acute radiation dermatitis in breast cancer patients treated with adjuvant radiotherapy. A randomised study of betamethasone versus two moisturizing creams. Radiother Oncol 2013;108:287-92.

29. Fuccio L, Guido A. Topical rectal beclomethasone dipropionate treatment for the prevention of radiationinduced bleeding. Gut 2012;61:1369.

30. Korinko A, Yurick A. Maintaining skin integrity during 
radiation therapy. Am J Nurs 1997;97:40-4.

31. Saag KG, Koehnke R, Caldwell JR, et al. Low dose longterm corticosteroid therapy in rheumatoid arthritis: an analysis of serious adverse events. Am J Med 1994;96:115-23.

32. Christoforou J, Karasneh J, Manfredi M, et al. World Workshop on Oral Medicine VII: Non-opioid pain management of head and neck chemo/radiationinduced mucositis: A systematic review. Oral Diseases 2019;25:182-92.

33. Chitapanarux I, Tungkasamit T, Petsuksiri J, et al. Randomized control trial of benzydamine $\mathrm{HCl}$ versus sodium bicarbonate for prophylaxis of concurrent chemoradiation-induced oral mucositis. Support Care Cancer 2018;26:879-86.

34. Liang L, Hu D, Liu W, et al. Celecoxib reduces skin damage after radiation: selective reduction of chemokine and receptor mRNA expression in irradiated skin but not in irradiated mammary tumor. Am J Clin Oncol 2003;26:S114-21.

35. Cheki M, Yahyapour R, Farhood B, et al. COX-2 in Radiotherapy: A Potential Target for Radioprotection and Radiosensitization. Curr Mol Pharmacol 2018;11:173-83.

36. Moore AH, Olschowka JA, Williams JP, et al. Regulation of prostaglandin E2 synthesis after brain irradiation. Int J Radiat Oncol Biol Phys 2005;62:267-72.

doi: $10.21037 /$ tro-20-46

Cite this article as: Huang WH, Liu SH, Huang GC, Su CW, Lee WJ, Chen FYS, Chen YJ. Preliminary study of therapeutic effect of parecoxib on severe radiation mucositis and dermatitis. Ther Radiol Oncol 2020;4:25.
37. El-Ghazaly MA, Nada AS, El-Hazek RM, et al. Effect of selective COX-2 inhibitor, celecoxib on adjuvantinduced arthritis model in irradiated rats. Int J Radiat Biol 2010;86:1079-87.

38. Lalla RV, Choquette LE, Curley KF, et al. Randomized double-blind placebo-controlled trial of celecoxib for oral mucositis in patients receiving radiation therapy for head and neck cancer. Oral Oncol 2014;50:1098-103.

39. Ghasemi A, Danesh B, Yazdani-Charati J, et al. Randomized Double-blind Placebo-controlled Trial of Celecoxib for the Prevention of Skin Toxicity in Patients Receiving Radiation Therapy for Breast Cancer. Antiinflamm Antiallergy Agents Med Chem 2018;17:57-67.

40. Debucquoy A, Roels S, Goethals L, et al. Double blind randomized phase II study with radiation +5 fluorouracil \pm celecoxib for resectable rectal cancer. Radiother Oncol 2009;93:273-8.

41. Ittichaikulthol W, Prachanpanich N, Kositchaiwat C, et al. The post-operative analgesic efficacy of celecoxib compared with placebo and parecoxib after total hip or knee arthroplasty. J Med Assoc Thai 2010;93:937-42.

42. Senzaki M, Ishida S, Yada A, et al. CS-706, a novel cyclooxygenase-2 selective inhibitor, prolonged the survival of tumor-bearing mice when treated alone or in combination with anti-tumor chemotherapeutic agents. Int J Cancer 2008;122:1384-90. 